group. Urinary free carnitine was reduced in valproate treated children with a significant increase in the ratio of bound to free carnitine. Carnitine supplementation is recommended.

Alterations of renal carnitine metabolism by valproic acid were studied in mice at the University of Santiago de Compostela, Spain (Camina MF et al. Neurology Sept 1991; $\underline{41}$ :1444-1448). Valproic acid induced a significant increase in renal clearance of acylcarnitine without affecting that of free carnitine, whereas other anticonvulsants increased clearance of free carnitine but not that of acylcarnitine.

Erratum. We thank Dr. J.M. Prats, Hospital de Cruces, Bilbao, Spain, for the correction of an error in the article concerning sodium valproate treatment of infantile spasms reviewed in Ped Neur Briefs Aug $1991 ; \underline{5}: 63$. The sentence should read as follows: "The hypsarrythmia EEG pattern was controlled after two weeks treatment with VPA 100 to 300 $\mathrm{mg} / \mathrm{Kg} /$ daily in $80 \%$ of patients."

\title{
EPILEPSY AND MENTAL RETARDATION
}

The cumulative risk of seizures and epilepsy was studied in a prospectively identified cohort of 221 children with mental retardation born between 1951 and 1955 in Aberdeen, Scotland and reported from the Montefiore-Einstein Epilepsy Management Center, Albert Einstein College of Medicine, Bronx, NY. Epilepsy had developed in $15 \%$ by 22 years of age. The cumulative risk of developing epilepsy varied from $5 \%$ in children with mental retardation to $75 \%$ in those with mental retardation associated with a postnatal brain injury. An apparent increased risk of epilepsy associated with severe mental retardation was attributed to the higher proportion of postnatal brain injury among this group. The cumulative risk of epilepsy at 22 years was $38 \%$ in children with mental retardation and cerebral palsy and $66 \%$ in those with postnatal injury. Of 20 children whose initial seizure was a febrile convulsion, $35 \%$ went on to develop epilepsy. The authors conclude that in the absence of associated disability or postnatal injury, the risk of epilepsy in the retarded population is low. (Goulden KJ, Shinnar S et al. Epilepsy in children with mental retardation: a cohort study. Epilepsia Sept/Oct 1991; 32:690-697).

COMMENT. This study is important in counseling and management of the child with mental retardation. The incidence of various seizure patterns known to be associated with mental retardation, particularly infantile spasms and Lennox-Gastaut, in the subgroups of retarded children would be of interest. West and Lennox-Gastaut syndromes are reviewed from the Children's Hospital, Ohio State University, Columbus, OH (Donat JF, Wright FS. Epilepsia July/Aug 1991; $\underline{32}$ :504509). 